\title{
Case report of a rare yeast Cyberlindnera fabianii fungemia in preterm twin neonates from North India: Diagnostic and therapeutic challenge
}

\author{
Nidhi Pandey ${ }^{1}$, Pradeep Paul ${ }^{2}$, Dhilip Kumar ${ }^{3}$, Ragini Tilak ${ }^{4}$ \\ From ${ }^{1}$ PhD Student, Department of Microbiology, Institute of Medical Sciences BHU, Varanasi, Uttar Pradesh, India, ${ }^{2}$ M.D/Senior Resident, \\ Department of Microbiology, Institute of Medical Sciences BHU, Varanasi, Uttar Pradesh, India, ${ }^{3}$ M.D/Senior Resident, Department of Pediatrics, \\ Institute of Medical Sciences BHU, Varanasi, Uttar Pradesh, India, ${ }^{4}$ M.D/Professor, Department of Microbiology, Institute of Medical Sciences BHU, \\ Varanasi, Uttar Pradesh, India
}

\begin{abstract}
Cyberlindnera fabianii infection has rarely been described in humans and only a few cases of human infections by this organism have been reported to date. Here, we present a case of premature twin neonates who were outborn and admitted for extremely low birth weight, early-onset sepsis, and patent ductus arteriosus. They were put on continuous positive airway pressure for 6 days and prolonged antibiotic course. Blood culture was positive for Candida sp. Antifungal drug fluconazole was added to the treatment regime which includes piperacillin-tazobactam and amikacin. Isolates were initially unidentified by conventional identification methods and showed reduced susceptibility to azoles. MALDI-TOF-MS identified the isolates as C. fabianii. Soon the neonates went into shock and cardiopulmonary arrest despite high ventilator support and died during treatment due to respiratory failure. This case report highlights C. fabianii as a rare fungal pathogen and emphasizes the importance of molecular methods such as matrix-assisted laser desorption/ ionization time-of-flight mass spectrometry (MALDI-TOF MS) in its accurate identification.
\end{abstract}

Key words: Neonates, Fungemia, Prematurity, MALDI-TOF-MS, Cyberlindnera fabianii, Fluconazole

$\mathrm{C}$ andida fabianii (teleomorph C. fabianii) was first described in 1964. Since then, species have been variedly described under the genus Hansenula or Pichia [1]. A new genus Lindnera in 2008 was proposed by Kurtzman et al., to include several previously known taxa on the basis of phenotypic and phylogenetic differences that also included C. fabianii (teleomorph: Lindnera fabianii) [2]. Until now, C. fabianii has not been associated with invasive animal or human disease, except in one report on prostatitis and urinary tract infection [3].

Here, we describe the case of a preterm neonate who was admitted for extremely low birth weight (ELBW) and prematurity with patent ductus arteriosus (PDA) and put on immediate antibiotic therapy and supportive measures. Blood culture was positive for Candida spp. which was correctly identified by MALDI-TOF-MS as C. fabianii. Both the neonate went into shock and cardiopulmonary arrest despite high ventilator support and died during treatment due to respiratory failure. In this present era of molecular advancements in the diagnosis, it has now become easier to identify the rare pathogens by robust techniques such as

\section{Access this article online}

Received - 17 August 2020

Initial Review - 01 September 2020

Accepted - 30 September 2020

DOI: $10.32677 /$ IJCR.2020.v06.i10.006
MALDI-TOF-MS and treat the challenging cases with accurate, reliable, and faster microbiological aid in diagnosis. This case throws light on the rare pathogenic yeast which a clinician should consider and keep in mind at the time of diagnosis which could be challenging to treat.

\section{CASE REPORT}

Outborn premature twin neonates (delivered at $35^{\text {th }}$ week of gestation), who were 48-h old, admitted in the pediatric emergency department with ELBW which was 900 grams for twin 1 and 950 grams for twin 2, early-onset sepsis, PDA, and fairly low APGAR score (5 for both). The mother was a 27 -year-old primigravida with a history of iron deficiency anemia.

On the date of admission, their hemogram revealed 400 leukocytes per $\mathrm{mm}^{3}$ with $24 \%$ neutrophils and hemoglobin $14.5 \mathrm{~g} / \mathrm{dl}$ for twin 1 and 500 leukocytes per $\mathrm{mm}^{3}$ with $26 \%$ neutrophils hemoglobin $13.4 \mathrm{~g} / \mathrm{dl}$ for twin 2 indicating that both neonates were neutropenic. Soon, the neonatal resuscitation was planned and the twin 1 was put on bubble continuous positive airway pressure for 6 days followed by the mechanical ventilation for 7 days and the twin 2, who had recurrent episodes of apnea,

Correspondence to: Ragini Tilak, Department of Microbiology, Institute of Medical Sciences BHU, Varanasi, Uttar Pradesh, India. E-mail: raginijain29@rediffmail.com

(C) 2020 The Author(s). This open access article is distributed under a Creative Commons Attribution (CC-BY) 4.0 license. 
was put on the mechanical ventilation for 4 days. Both of them received i.v. fluids, i.v. antibiotics (piperacillin-tazobactam at a dose of $80 \mathrm{mg} / \mathrm{kg} /$ dose IV every $6 \mathrm{~h}$ and amikacin at a dose of $15 \mathrm{mg} / \mathrm{kg} \mathrm{IV} \mathrm{q} 24 \mathrm{~h}$ ) and also received i.v. paracetamol for PDA.

Blood samples were collected from both the neonates from two different sites by heel prick of the right and left medial plantar surface and were sent for culture and sensitivity. Blood culture revealed the growth of Candida spp. in all the four biphasic blood culture bottles of both the neonates, for which fluconazole was started.

On day 16, twin 1 went into shock and was not maintaining saturation even with high ventilator support, and went into cardiopulmonary arrest. despite giving cardiopulmonary resuscitation the neonate, could not be revived and finally died due to respiratory failure. After $48 \mathrm{~h}$ of birth, twin 2 developed gastrointestinal hemorrhage due to disseminated intravascular coagulation and was desaturating, followed by bradycardia and could not be revived despite all the available measures.

Both the yeast species were found to be sensitive to fluconazole, voriconazole (MIC $0.5 \mu \mathrm{g} \mathrm{ml}^{-1}$ ), and amphotericin B (MIC $0.25 \mu \mathrm{g} \mathrm{ml}^{-1}$ ). The isolates were sent to the Department of Microbiology, Post Graduate Institute of Medical Education and Research, Chandigarh, India, for confirmation of the species. Both the isolates were identified as C. fabianii (Figs. 1 and 2) by MALDI-TOF M S with a BRUKER value of 2.50 and 2.23

\section{DISCUSSION}

C. fabianii has rarely been described in humans and only a few cases of human infections by this organism have been reported to date $[4,5]$. Failure of the conventional methods to identify this Candida spp. led us to perform molecular identification of the yeast. It was eventually identified as C. fabianii (also known as Lindnera fabianii or Pichia fabianii). As commercial yeast identification and diagnostic kits (e.g., API 20CAUX, ID32C, and Vitek 2) used in routine practice have a poor ability to identify C. fabianii, this organism has also been misidentified as Pichia anomala or Candida utilis in other [6,7]. In 2013, Yun et al. reported a case of $C$. fabianii from the blood culture of a 47-yearold woman which was identified initially as $C$. utilis by Vitek 2 . C. fabianii is pathogen of low and is the teleomorphic form of C. fabianii, which was described [8].

In recent years, MALDI TOF MS has proven a powerful technique that circumvents many challenges posed by other conventional techniques and is being adapted for rapid identification of fungi due to its high performance and less time requirement $[9,10]$. The technique relies on the generation of microorganism "protein fingerprints" that are compared to reference spectra in a well-characterized library. It has now reached a level at which the identification of complex mass spectra in databases has become much easier in a standardized fashion. Compared to biochemical procedures involving overnight growth, this is a highly time-saving procedure and can significantly speed up diagnostic processes. Furthermore, the technique is inexpensive and does not require experienced personnel.

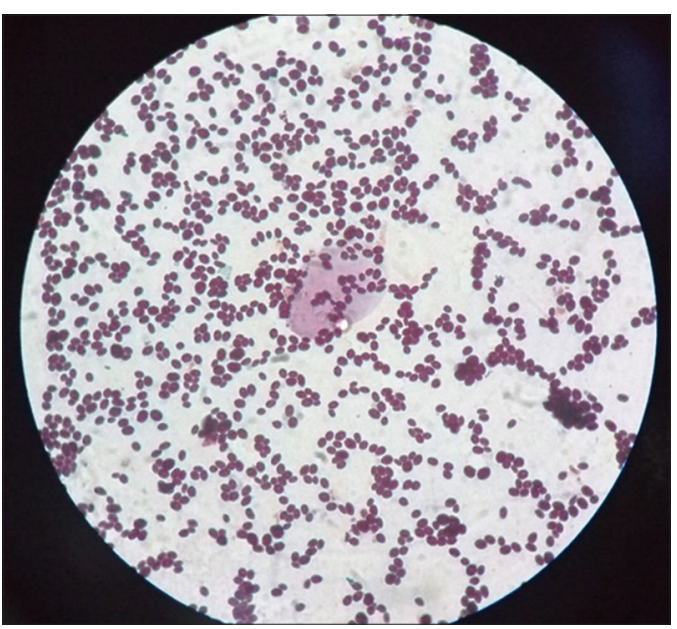

Figure 1: Gram stain image of Cyberlindnera fabianii

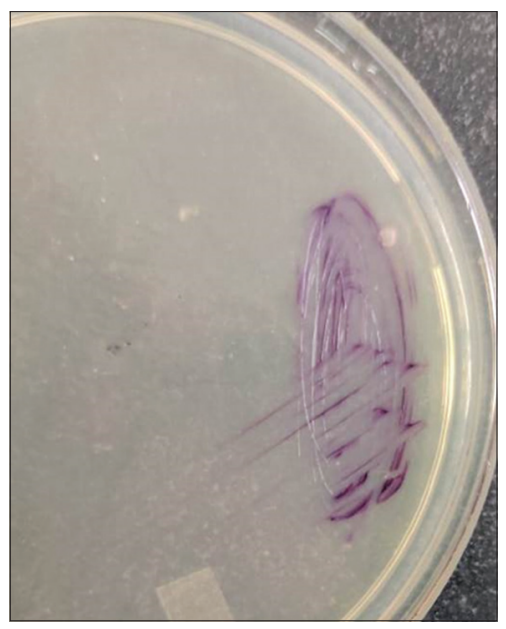

Figure 2: Purple color of yeast colonies on CHROM agar

Here, we present a case report that describes the isolation of C. fabianii from the blood culture of 48-h old ELBW premature twin neonates. Till now there are only five cases of $C$. fabianii fungemia have been reported worldwide which includes four adult patients $[6,8]$ and three neonates $[7,11,12]$. To the best of our knowledge, in earlier cases, isolation of $C$. fabianii has only been reported from blood specimens and only infrequently. Risk factors associated with Candida infection are exposure to broad-spectrum antimicrobial agents, indwelling vascular catheters, prior surgery, and cancer chemotherapy. In this case report, the risk factors identified were ELBW, prematurity, indwelling catheters, and febrile neutropenia. As the isolated strain was sensitive to amphotericin $\mathrm{B}(\mathrm{MIC}=0.25 \mu \mathrm{g} / \mathrm{ml})$ showing discordance from previous cases, one from China, in which P. fabianii isolated from the blood sample of a premature infant, had shown resistance to itraconazole and amphotericin B [12]. Another case report from Korea described clinical resistance of $L$. fabianii to amphotericin $\mathrm{B}$, although the in vitro MIC value was $0.5 \mu \mathrm{g} \mathrm{ml}^{-1}[8]$ and one case report from India described in urine isolate that was resistant to amphotericin $\mathrm{B}$ (MIC $>4 \mu \mathrm{g} \mathrm{ml}^{-1}$ ). Thus, it is important to study antifungal susceptibility, along with speciation of the Candida isolates.

The limitations of our study were as follows: (i) It could not be confirmed whether $C$. fabianii was a true pathogen and causes 
of mortality or an incidental finding, as a repeat sample was not available; (ii) the source of infection could not be traced. As the neonates were febrile at the time of admission, the infection was not acquired nosocomially from the present institution; however, it might have been acquired during the previous hospitalization.

\section{CONCLUSION}

To the best of our knowledge, we are reporting the first case of isolation of $C$. fabianii from a blood specimen from North India. We also emphasize the role of MALDI-TOF-MS in confirming the identity of uncommon fungal isolates, as correct identification is important for epidemiological purposes. Clinicians should consider the possibility of rare pathogenic yeast especially in neonates at the time of diagnosis which could be challenging to treat and may lead to fatal outcome.

\section{ACKNOWLEDGMENT}

Department of Science and Technology, New Delhi is gratefully acknowledged. We are thankful to the Mycology Division, Post Graduate Institute of Medical Education and Research, Chandigarh, India, for the confirmatory identification of C. fabianii.

\section{REFERENCES}

1. Kurtzman CP. Lindnera Kurtzman, Robnett, and BasehoarPowers In: Kurtzman CP, Fell JW, Boekhout T, editors. The Yeasts, a Taxonomic Study. $5^{\text {th }}$ ed. Amsterdam: Elsevier; 2008. p. 521-43.

2. Kurtzman CP, Robnett CJ, Basehoar-Powers E. Phylogenetic relationshipsamong species of Pichia, Issatchenkia and Williopsis determined from multigene sequence analysis, and the proposal of Barnettozyma gen. nov., Lindnera gen. nov. and Wickerhamomycesgen. nov. FEMS Yeast Res 2008;8:939-54.

3. Dooley DP, Beckius ML, McAllister CK, Jeffery BS. Prostatitis caused by Hansenula fabianii. J Infect Dis 1990;161:1040-1.

4. Hazen KC, Theisz GW, Howell SA. Chronic urinary tract infection due to Candida utilis. J Clin Microbiol 1999;37:824-27.

5. Grlic L, Missoni MA, Skaric E, Babic VI, Svetec IK. Candida utilis candidaemia in neonatal patients. J Med Microbiol 2011;60:838-41.

6. Gabriel F, Noel T, Accoceberry I. Lindnera (Pichia) fabianii blood infection after mesenteric ischemia. Med Mycol 2012;50:310-4.

7. Grenouillet F, Millon L, Chamouine A, Thiriez G, Schulze O, Leroy J. Pichia fabianii fungemia in a neonate. Pediatr Infect Dis J 2010;29:191-5.

8. Yun JW, Park KS, Ki CS, Lee NY. Catheter-related bloodstream infection by Lindnera fabianii in a neutropenic patient. J Med Microbiol 2013;62:922-5.

9. Jindal N, Arora S, Dhuria N. Cyberlindnera (Pichia) fabianii infection in a neutropenic child: Importance of molecular identification case report. J Med Microbiol 2015;3:1-3.

10. Ceballos-Garzón A, Cortes G, Morio F, Zamora-Cruz EL, Linares MY, Ariza BE, et al. Comparison between MALDI-TOF MS and MicroScan in the identification of emerging and multidrug resistant yeasts in a fourth-level hospital in Bogotá, Colombia. BMC Microbiol 2019;19:106.

11. Bhally HS, Jain S, Shields C, Halsey N, Cristofalo E, Merz WG. Infection in a neonate caused by Pichia fabianii: Importance of molecular identification. Med Mycol 2006;44:185-7.

12. Wu Y, Wang J, Li W, Jia H, Che J, Lu J, et al. Pichia fabianii blood infection in a premature infant in China: Case report. BMC Res Notes 2013;6:77.

Funding: None; Conflicts of Interest: None Stated.

How to cite this article: Pandey N, Paul P, Kumar D, Tilak R. Case report of a rare yeast Cyberlindnera fabianii fungemia in preterm twin neonates from North India: Diagnostic and therapeutic challenge. Indian J Case Reports. 2020;6(10):563-565. 